\title{
Clinical Data Update System
}

National Cancer Institute

\section{Source}

National Cancer Institute. Clinical Data Update System. NCI Thesaurus. Code C19992.

A system to standardize and streamline data reporting and reduce administration needs.

This system achieves rapid, accurate, and secure capture of clinical trial data with minimal effort. Successfully captures the majority of trial data within hours of submission.

(Bypass Budget 2001 \& 2002) 